\title{
FEM Simulation and Optimization of the Electromechanical Behavior of a Variable Superconducting Niobium Capacitor
}

\author{
T. Zöpfl*, W. Bedyk, F. Wittmann, G. Schrag, G. Wachutka and M. Schicke1 \\ Institute for Physics of Electrotechnology, Munich University of Technology, \\ Arcisstr. 21, 80290 München, Germany \\ ${ }^{1}$ Institut de RadioAstronomie Millimétrique (IRAM), \\ 300 rue de la piscine, 38406 St. Martin d'Hères, France
}

(Received September 18, 2007; accepted March 27, 2008)

Key words: tunable Nb capacitors, electromechanical FEM simulation, intrinsic stress, whitelight interferometry

Superconducting $\mathrm{GHz}$ electronic circuits are frequently used in radio astronomy in view of their low electrical losses and, thus, their minimal noise level. The performance of these circuits can be significantly improved by applying tunable capacitors, which can be realized as electrostatically actuated, micromechanical bridges made of superconducting niobium ( $\mathrm{Nb})$. To analyze the electromechanical behavior of such devices and the intrinsic stress gradient induced during the fabrication process, finite element (FE) models of the bridges and of dedicated test structures have been built. Furthermore, with a view to calibrating and validating the underlying physical model, the topography of bridges under zero voltage as well as under electrostatic actuation has been investigated by white-light interferometry. On this basis, a semianalytical model was derived, which allows a simple estimation of the pull-in voltage. Design studies based on the calibrated model have been successfully carried out to optimize devices with respect to the required specifications for their application.

\section{Introduction and Description of Problem}

Variable tuning elements for high-frequency applications are of increasing interest in the field of radio astronomy, where low electrical losses, extremely high sensitivity, and minimal noise level are inevitable requirements for signal detection. ${ }^{(1)}$ Devices manufactured from superconducting materials meet these demands and are well established in this field. ${ }^{(2)}$

Variable capacitors, so-called varactors, represent one important class of tunable circuit elements. They allow the continuous tuning of capacitance and, therefore, are

${ }^{*}$ Corresponding author: e-mail: teresa.zoepfl@tep.ei.tum.de 
widely applied in wireless communication, radar, and frequency- converting systems, ${ }^{(3-5)}$ where they serve as basic elements for adjusting the operating range of variable filters, phase shifters, and transceivers.

Using current microfabrication technology, veractors are increasingly realized as electrostatically actuated micromechanical switches, ${ }^{(6,7)}$ which exhibit very low electrical losses compared with other varactor principles. Employing tunable microelectro-mechanical (MEMS) capacitors has made it possible to significantly improve the performance of superconducting $\mathrm{GHz}$ electronic circuits for radio-astronomic applications. ${ }^{(8)}$ They are typically utilized for adaptive impedance matching between the tunnel diode and antenna or for direct integration in superconductor-isolatorsuperconductor (SIS) mixers, where they act as tunable band-stop filters of undesired artificial celestial signals.

To this end, Schicke and coworkers ${ }^{(9,10)}$ developed a tunable microcapacitor (varactor) made of superconducting niobium (Fig. 1). Designed as an electrostatically actuated micromechanical bridge, it can be fabricated using the same technology as that used for other superconducting high-frequency devices and, thus, can be easily integrated into the respective electronic $\mathrm{GHz}$ circuitry within the same process steps.

The operating principle of tunable $\mathrm{Nb}$ microcapacitors is illustrated in Fig. 2. The bridges can be deflected by applying an appropriate DC voltage between the movable part and the fixed ground electrode. Thus, the gap underneath the bridge, and thereby the capacitance of the device, can be precisely tuned.

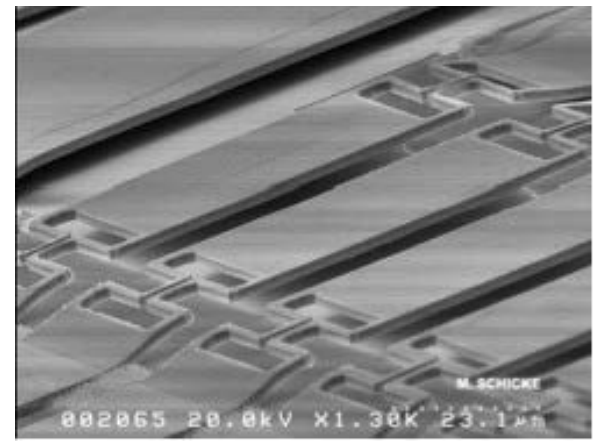

Fig. 1. Scanning electron micrograph (SEM) showing a series of Nb microbridges suspended by meander-shaped hinges. ${ }^{(1)}$

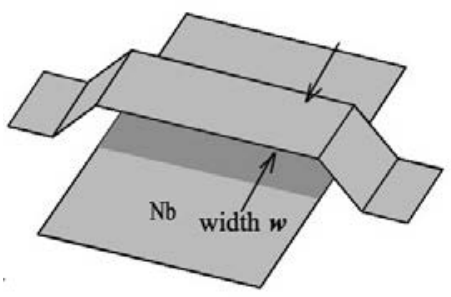

(a)

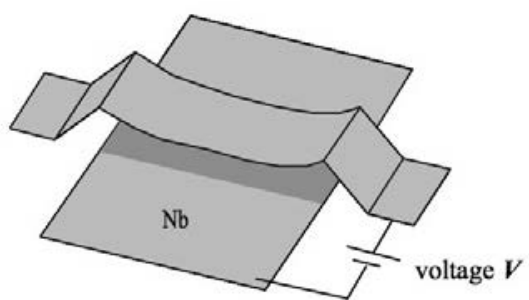

(b)

Fig. 2. Operating principle of the Nb varactor: bridge (a) without and (b) with applied voltage $V$. 
To reduce the stiffness of the mechanically movable part and, thus, to reduce the actuation voltage of the device, the suspensions of the bridges are realized as meanders, as schematically shown in Fig. 3. Typical geometrical dimensions of the bridges and meanders are listed in Table 1 . The length of the rectangular bridge varies between $50 \mu \mathrm{m}$ and $200 \mu \mathrm{m}$, and the width varies between $50 \mu \mathrm{m}$ and $1080 \mu \mathrm{m}$. The meanders are at least $2 \mu \mathrm{m}$ wide, the thickness of the Nb bridge varies from $1 \mu \mathrm{m}$ to $2 \mu \mathrm{m}$, and the capacitance under zero voltage lies between $5 \mathrm{fF}$ and a few hundred $\mathrm{fF}$.

The Nb bridges are fabricated by a surface micromachining MEMS process. The superconducting $\mathrm{Nb}$ is sputtered onto the substrate to form the ground electrode. Subsequently, photoresist is deposited, which acts as a sacrificial layer to define the bridge profile. Then a second layer of $\mathrm{Nb}$ is sputtered on the photoresist, structured to form the rectangular bridge region and meander suspensions, and released by removing the sacrificial layer by etching. During the sputtering process, differential intrinsic stress is induced in the Nb layer, which causes a deformation of the bridges already under unloaded conditions.

This study comprises the experimental characterization of such variable $\mathrm{Nb}$ capacitors by white-light interferometry and their predictive simulation using calibrated numerical (FE) and analytical models. To this end, we derived a physically-based fully coupled electromechanical FE simulation model, which has been carefully verified

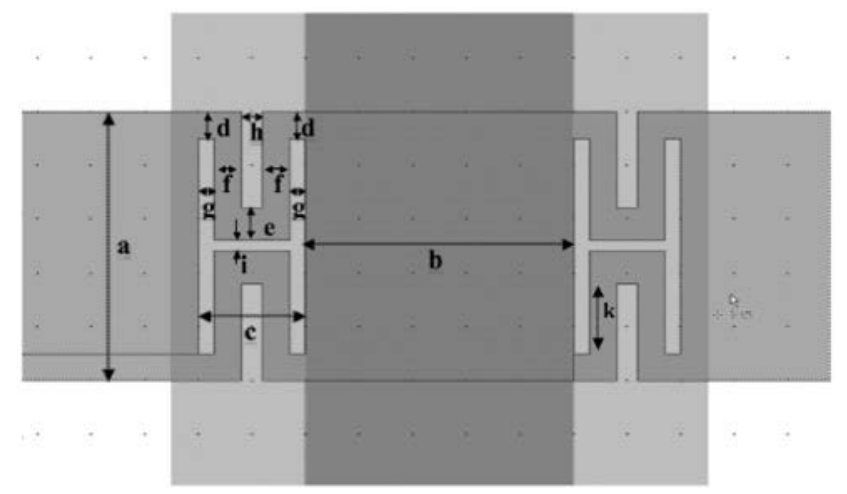

Fig. 3. Schematic view of the microbridge and the meander suspensions. EE mplar values of dimensions $a$ to $i$ are given in Table 1 .

Table 1

Exemplar values (in $\mu \mathrm{m}$ ) of the geometrical dimensions $a$ to $i$ of the bridge and meanders (see Fig. 3).

\begin{tabular}{rrrrrrrr}
\hline \multicolumn{1}{c}{$a$} & \multicolumn{1}{c}{$b$} & \multicolumn{1}{c}{$d$} & \multicolumn{1}{c}{$e$} & $f$ & $g$ & $h$ & $i$ \\
\hline 50 & 50 & 4 & 2 & 2 & 5 & 6 & 2 \\
100 & 100 & 8 & 12 & 6 & 3 & 2 & 2 \\
100 & 160 & 12 & 9 & 4 & 6 & 5 & 2 \\
\hline
\end{tabular}


and calibrated by measurements. It enables the reliable and predictive simulation of the device behavior, in particular, the detailed analysis of all stress effects and the identification of the relevant parameters; hence, it can serve as a tool for optimizing the design of bridges and suspensions, particularly with respect to minimizing the pullin voltage. Additionally, we supplemented the detailed FE model with an easy-touse semianalytical model for the rapid calculation of the pull-in voltage. However, this simplified approach also includes a reduced numerical 3D-FE model as the core, because a purely analytical model based on conventional beam theory ${ }^{(11)}$ cannot properly describe the elastomechanical properties of the meander-shaped suspensions. In contrast to previously reported analytical models, ${ }^{(11-13)}$ our approach gives a correct description of the elastic behavior of the deformable bridge area in conjunction with that of the meander suspensions.

The organization of our paper is as follows: In $\S 2$ we describe the experimental characterization of the deformed microbridges by white-light interferometry (WLI). Then, in $\S 3$, we discuss dedicated test structures that we developed and analyzed to extract the value and gradient of the intrinsic stress. Section 4 deals with the electromechanical FE simulation model of the microbridges, its calibration, and its verification employing the measured data. This model is used in $\S 5$ to perform design studies and sensitivity analyses of the device. Finally, in $\S 6$, we discuss the validity of the simplified analytical and semianalytical models, and give a summary in $\S 7$.

\section{Experimental Characterization}

The topography of bridges was analyzed by WLI with an amplification of 1.25 to 20 (Veeco NT 1100). This characterization method utilizes white light with a short coherence length, which enables the contactless topographical analysis of microstructures with a high vertical resolution of 5-6 $\mathrm{nm}$ and a lateral resolution of about $2 \mu \mathrm{m}$.

Two types of measurement were employed. First, the devices were characterized without an actuation voltage to study the effect of intrinsic stress on the initial shape. Second, a variable DC voltage was applied to the bridge layer, allowing us to extract electromechanical parameters, such as the initial gap height and the stiffness of the meanders. The measurements were carried out on devices with rectangular and square bridge areas and a Nb layer thickness of $1.5 \mu \mathrm{m}$. The WLI measurements without an actuation voltage revealed the overall topography of the microbridges including the meander hinges (Fig. 4) as well as the details of the deformation of only the bridge area (Fig. 5). Figures 4 and 5 show exemplar results of the WLI measurements. Here, light colored regions refer to structural parts that are elevated, in contrast with darker device regions.

Measurements of only the bridge area (Fig. 5) indicate that the deformation profile is not the same for all bridge geometries. While square bridges show a cylindrical, saddlelike deflection (Fig. 5(b)), rectangular bridges are bent symmetrically with respect to the center point of the plate (Fig. 5(a)). The edges of the bridge are bent upwards for both geometries. The maximum value of the center deflection lies between $0.2 \mu \mathrm{m}$ and 0.3 $\mu \mathrm{m}$ for both bridge geometries. 


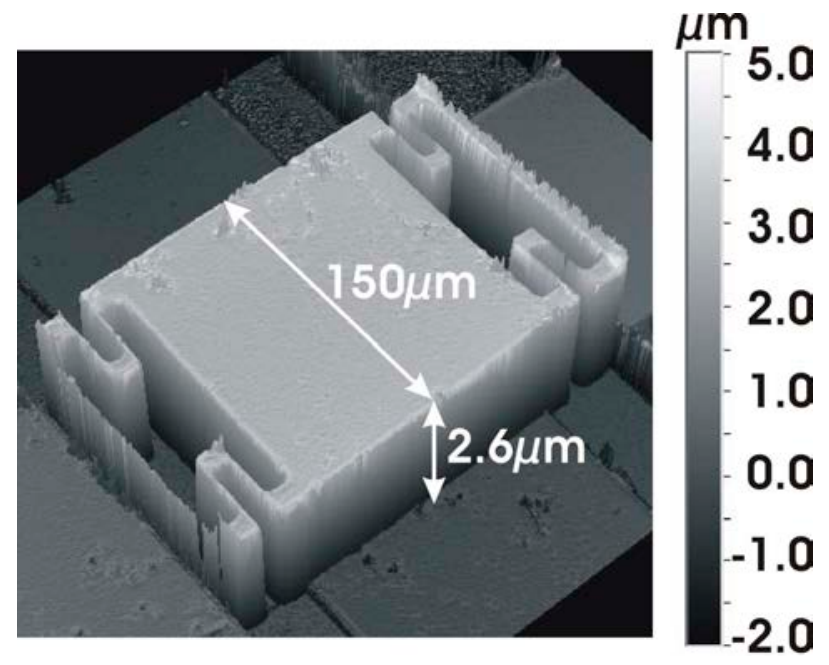

Fig. 4. Interferometric measurement of a microbridge with a gap height of $2.6 \mu \mathrm{m}$.

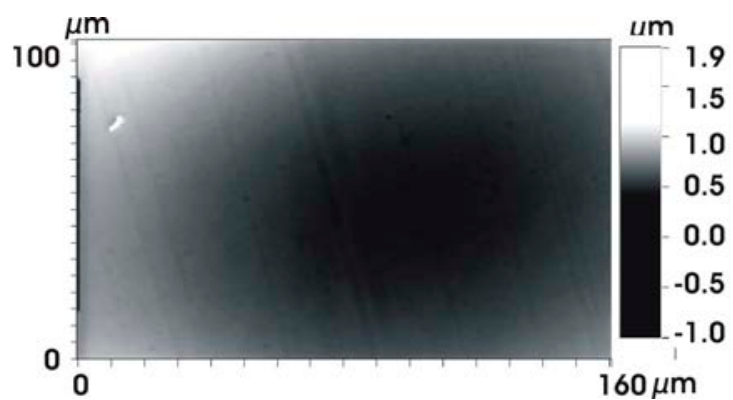

(a)

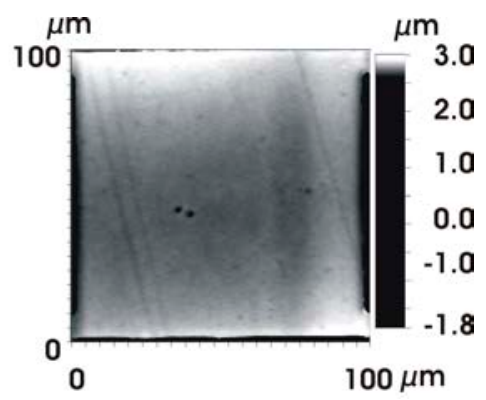

(b)

Fig. 5. Interferometric measurements of the bridge surface: deflection of (a) rectangular and (b) square bridge areas.

From WLI measurements with a variable DC voltage applied, the pull-in voltage of the devices could be extracted by stepping up the applied DC voltage to the pull-in point. These results were compared with $\mathrm{C}-\mathrm{V}$ measurements, which had been carried out prior to this study. For both data sets, the values of the pull-in voltage agree within a maximum variance of $6 \%$. This also indicates the accuracy of the WLI measurements and allows us to now study the bending shape of the bridges under electrostatic actuation in detail, in particular, how the middle part of the bridge is deformed relative to the edges of the plate. This, in turn, constitutes valuable information for the verification of the simulation model.

In contrast to electrical measuring methods, the optical characterization provides direct access to topographical and electromechanical data such as the exact device geometry, bending shape, gap height, and pull-in voltage. Knowing these parameters is necessary to derive, calibrate, and verify the accuracy and predictivity of the simulation model. 


\section{Intrinsic Stress Investigations}

The intrinsic stress inside the Nb layer resulting from the fabrication process becomes evident in the initial deflection of the bridge, which may affect its function and performance. Therefore, prior to performing detailed theoretical and experimental studies of the operational behavior, it is advisable to investigate this undesired but unavoidable phenomenon and to determine the value and gradient of the intrinsic stress inside the Nb layer of the bridge. Unfortunately, the stress cannot be measured directly using the well-known wafer bow method, since the photoresist, which forms the sacrificial layer, is not spread uniformly over the substrate. Therefore, the measured stress value for the non-released bridges would also include the stress inside the sacrificial layer, which cannot be corrected by simple calculations to obtain the effective value for the Nb layer. Moreover, stress gradients, which are primarily the cause of the deformation of the bridges, can only be determined by this measuring technique if it is applied repeatedly during the deposition process, which is not possible using the given technology. Therefore, we decided to determine the stress gradient by means of special test structures, which were fabricated for this purpose, optically characterized, and subsequently numerically analyzed using a FE model. The test structures consisted of a $\mathrm{Nb}$ beam with a length of approximately $600 \mu \mathrm{m}$, a width of $150 \mu \mathrm{m}$, and a thickness of $720 \mathrm{~nm}$. When one side of these beams is released, they roll up into a loop with a diameter of about $200 \mu \mathrm{m}$, as measured by WLI. This implies a stress gradient along the depth of the layer with compressive stress at the bottom of the layer (Fig. 6(a)). The FE model of these test structures is built of three dimensional shell elements, exploits symmetry reduction, and uses the following material parameters (used also for all subsequent FE models): $E=1.05 \times 10^{11} \mathrm{MPa}, v=0.38$, and $\alpha=7.3 \times 10^{-6} 1 / \mathrm{K}$, where $E$

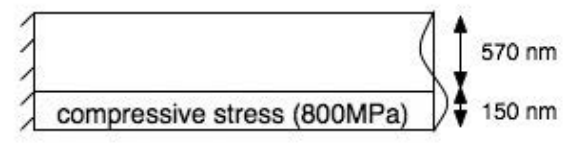

(a)

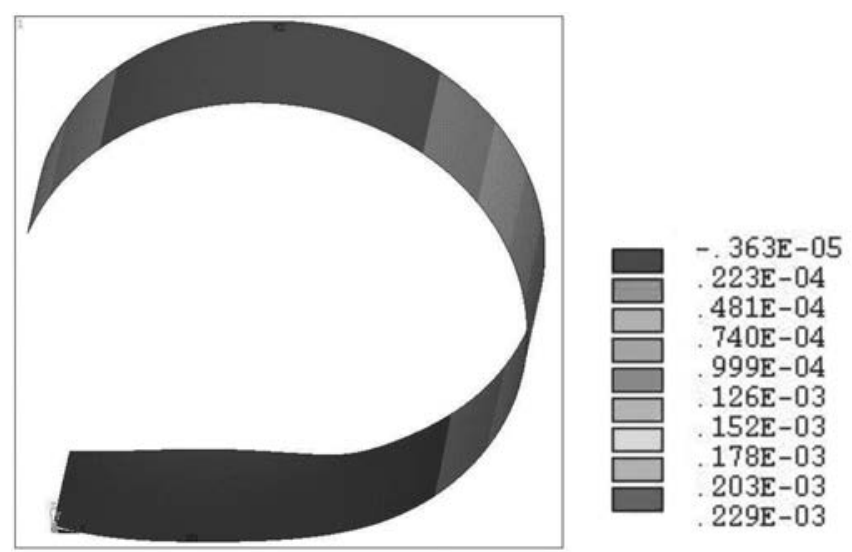

(b)

Fig. 6. Simulation of a test structure used to extract the value of the stress gradient within the $\mathrm{Nb}$ layer: (a) schematic view of the stress gradient inside the layer and (b) simulation result based on a $3 \mathrm{D}$ analysis (width of the beam is $150 \mu \mathrm{m}$ ). 
denotes Young's modulus, $v$ is Poisson's ratio, and $\alpha$ is the thermal expansion coefficient. The results of nonlinear buckling analysis revealed that a compressive stress of 800 $\mathrm{MPa}$ applied to the first $150 \mathrm{~nm}$ of the layer is necessary to reproduce the experimental findings (Fig. 6(b)).

\section{Simulation Model of the Microbridges}

\subsection{Finite element model}

For the numerical analysis of the $\mathrm{Nb}$ bridges we used the commercial FE simulator ANSYS ${ }^{(14)}$ and employed three different types of structural finite elements for comparison: 2D shell elements, 3D solid elements, and 3D shell elements. The internal stress distribution inside the $\mathrm{Nb}$ layer was emulated by applying a temperature body load distributed along the layer thickness, and the electrostatic actuation of the bridge was taken into account using special transducer elements (trans126), which allow the modeling of electromechanically coupled problems up to the pull-in point employing the direct matrix coupling method. ${ }^{(14)}$ In this theoretical approach, the "through" variables, electrical force $f$ and current $i$, are related to the "across" variables, voltage $u$ and displacement $x$, as follows:

$$
f=\frac{1}{2} \frac{\mathrm{d} C}{\mathrm{~d} x} u^{2}
$$

and

$$
i=C w+\frac{\mathrm{d} C}{\mathrm{~d} x} u v
$$

where $C$ is the electric capacitance, $v$ is the velocity of the gap opening $\mathrm{d} x / \mathrm{d} t$, and $w$ is the voltage rate $\mathrm{d} u / \mathrm{d} t$. The coupling of the mechanical and electrical domains is realized through the off-diagonal entries in the stiffness and damping matrices, which represent the self-consistently coupled system in linearized form:

$$
\left\{\begin{array}{l}
i_{0} \\
f_{0}
\end{array}\right\}+\left[\begin{array}{ll}
K_{u и} & K_{u x} \\
K_{x и} & K_{x x}
\end{array}\right]\left\{\begin{array}{l}
\mathrm{d} u \\
\mathrm{~d} x
\end{array}\right\}+\left[\begin{array}{ll}
D_{u и} & D_{u x} \\
D_{x u} & D_{x x}
\end{array}\right]\left\{\begin{array}{l}
\mathrm{d} w \\
\mathrm{~d} v
\end{array}\right\}=\left\{\begin{array}{l}
i \\
f
\end{array}\right\},
$$

where $i_{0}$ and $f_{0}$ denote the current and the force at the operating point, respectively. The entries of the matrices can be calculated from $K_{u u}=\partial i / \partial u$ and so forth. ${ }^{(15)}$ Since eq. (3) constitutes a linear system, only one computational iteration is necessary.

The electromechanical simulations of the microbridges were carried out using four different types of finite elements, and the resulting $\mathrm{C}-\mathrm{V}$ curves were compared with each other (Fig. 7). The 2D shell elements served as a reference for the 3D shell and 3D solid elements, which provided accurate results, in contrast to the model that uses beam elements for modeling the meander suspensions. This latter finding is corroborated by the analytical investigations described in $\S 6$. 


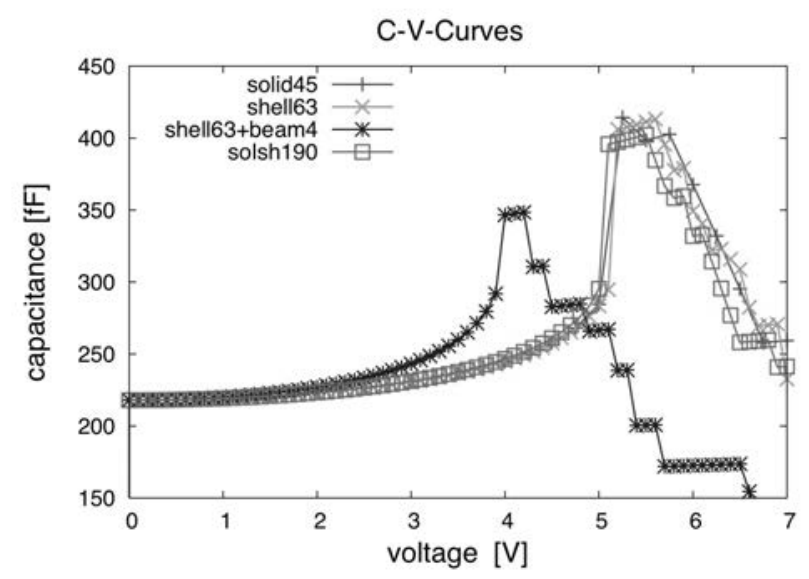

Fig. 7. FE simulation of C-V curves: comparison of simulation results obtained from different types of finite elements.

Since an internal stress gradient cannot be adequately represented by 2D shell elements, 3D shell and 3D solid elements, both of which give comparatively accurate results, were used for subsequent simulations.

\subsection{Calibration of the FE model}

The electromechanical behavior of the bridge is very sensitive to the initial gap height between the bridge and the ground electrode. The exact value of this crucial parameter, however, is not exactly known after fabrication, since the bridge changes its shape after it has been released from the sacrificial resist layer. Therefore, the gap height was extracted from WLI measurements of electrostatically actuated devices. From these measurements the exact value was determined by subtracting the data sets, which were obtained under zero voltage and after reaching the pull-in point of the bridge. The value of the gap height was about $2.6 \mu \mathrm{m}$ (Fig. 8).

Before releasing the bridge, profilometric measurements of the bridge profile were made, revealing a value of $2.89 \mu \mathrm{m}$ for the gap underneath the bridge. This agrees well with the interferometric measurement, because the deflection of the bridge due to intrinsic stress, which was $0.3 \mu \mathrm{m}$, does not become apparent until the bridge is released and, therefore, has to be subtracted from the value obtained from the profilometer data.

\subsection{Effect of intrinsic stress on the device performance}

The value of $800 \mathrm{MPa}$ for the intrinsic stress, which was previously obtained from test structures (see $\S 3$ ), was used in the above-mentioned FE model of a capacitor subjected to a distributed temperature body load. We calculated the deformation of the center point to be about $2 \mu \mathrm{m}$ for a bridge thickness of $1.5 \mu \mathrm{m}$ and a gap height of $2.6 \mu \mathrm{m}$ (see Fig. 9). 


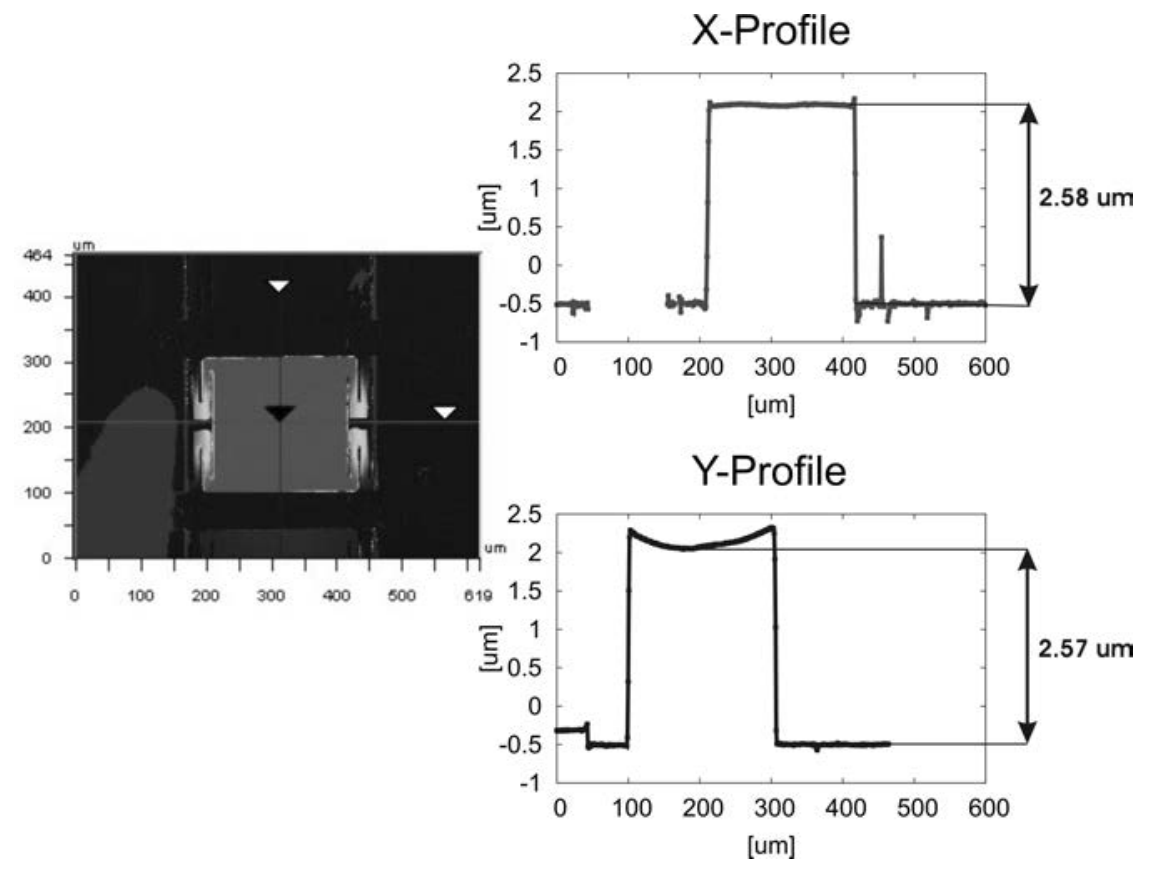

Fig. 8. Interferometric data used for the extraction of the gap height.

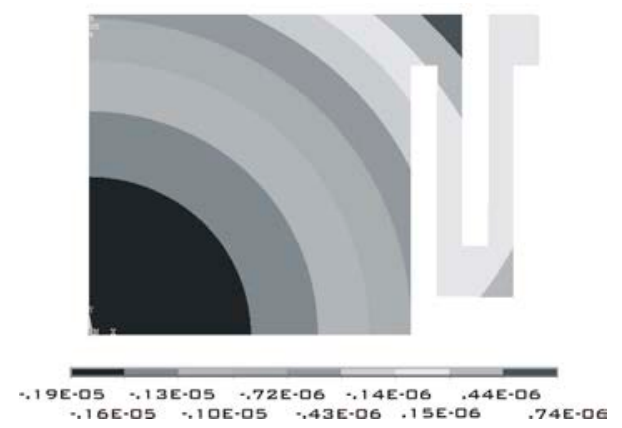

Fig. 9. FE simulation: displacement in z-direction for $800 \mathrm{MPa}$ intrinsic stress in the first $150 \mathrm{~nm}$ of the bridge length. The maximal deflection at the center is about $2 \mu \mathrm{m}$.

The WLI measurements, however, yield a deformation of only $0.3 \mu$ m, which would imply a pre-stress of $100 \mathrm{MPa}$ along the first $100 \mathrm{~nm}$ of the Nb layer. This discrepancy might originate from different stress relaxation mechanisms occurring in microstructured devices compared with the large-area test structures.

In addition, further investigations (simulations of the fundamental mode for varying intrinsic stress values along the first $100 \mathrm{~nm}$ of the bridge layer) revealed that the effect of the intrinsic stress on the dynamical behavior of the bridges is only marginal (Fig. 10). 


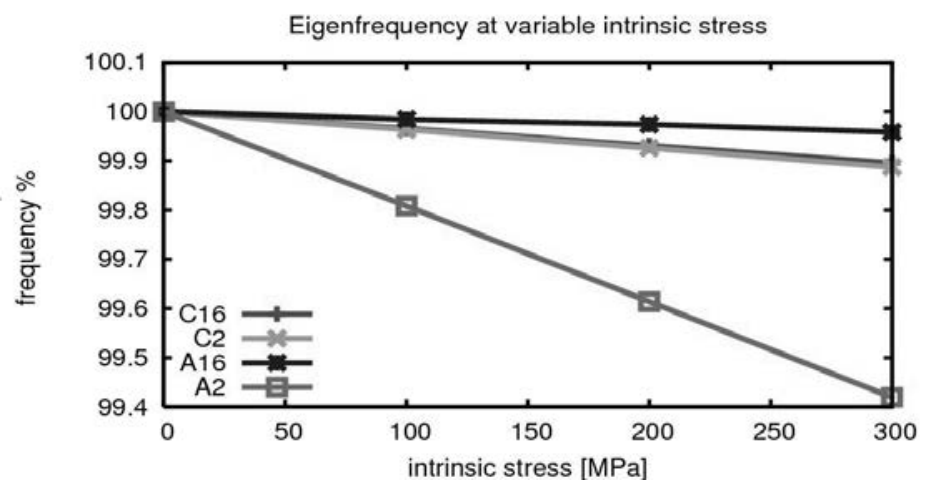

Fig. 10. Variation of the eigenfrequency of different bridge and meander geometries due to intrinsic stress in the first $100 \mathrm{~nm}$ of the bridge layer.

The investigated eigenfrequencies vary by no more than $0.6 \%$ for different bridge areas (A: $a=b=50 \mu \mathrm{m}, \mathrm{C}: a=b=100 \mu \mathrm{m}$ ) and meander geometries (2: high meander stiffness, 16: low meander stiffness).

This finding suggests that the initial deformation due to the internal stress gradient does not necessarily have to be included in the mechanical simulation model of the device.

\subsection{Verification of the FE model}

To verify the FE model of the varactors, electromechanical simulations of the measured $\mathrm{C}-\mathrm{V}$ curves were carried out, first without including the effects of intrinsic stress. As mentioned above, the pull-in voltages determined from the interferometric measurements and those from previous $\mathrm{C}-\mathrm{V}$ measurements are in good agreement. These pull-in voltages are also reproduced by the simulation when the gap height extracted from the interferometric experiment is used for model calibration. If the gap is chosen according to the zero voltage capacitance obtained from previous $\mathrm{C}-\mathrm{V}$ measurements $(\sim 1.7 \mu \mathrm{m})$, the value of the pull-in voltage differs by nearly a factor of two from the measured data, which indicates an uncertainty in the capacitance measurements (offset due to parasitic capacitances). Figure 11 shows a comparison of $\mathrm{C}-\mathrm{V}$ measurements and FE simulation results obtained for different values of the gap height. The results achieved using the calibrated model agree very well with the measured characteristics, whereas the difference in the total capacitance is due to systematic errors (offset) in the capacitance measurements.

In addition, the deformation of the bridge under an applied DC voltage was investigated by FE simulations and compared with interferometric data. An exemplar comparison of a measured cross-sectional deflection profile with simulated values of the z-deflection for an applied voltage of $47 \mathrm{~V}$ is shown in Fig. 12 (analogous to the bridge $\mathrm{X}$-profile depicted in Fig. 8). The simulated displacement of the bridge area is in good 


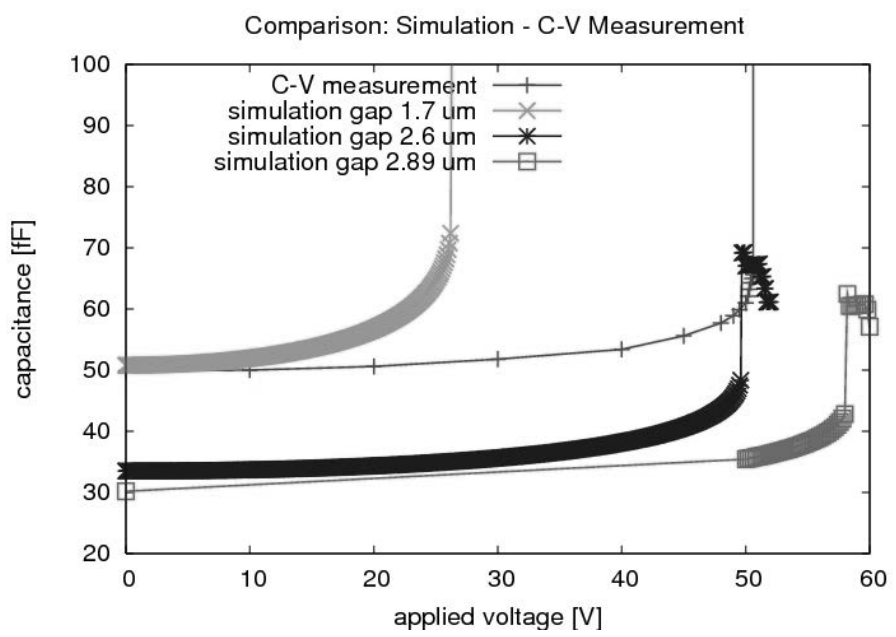

Fig. 11. Comparison of $\mathrm{C}-\mathrm{V}$ measurement and simulation results for different values of the gap height.

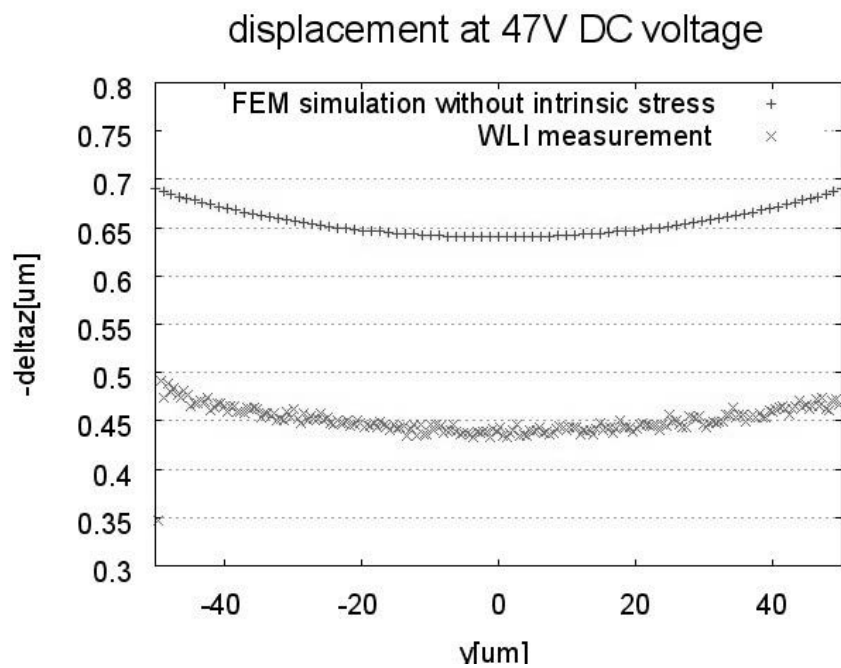

Fig. 12. Measurement and simulation of the displacement of the bridge area under an applied DC voltage (analogous to the X-profile in Fig. 8).

agreement with the measured data, even if the initial stress-induced deformation is not taken into account in the FE model. This means that the overall vertical displacement of the bridge is almost completely insensitive to the initial bridge deflection, which confirms the conclusion that intrinsic stress can be considered a minor effect in these devices and need not be included in the design studies discussed below. 


\section{Optimization Studies and Sensitivity Analysis}

\section{$5.1 C$-V curves}

The calibrated and verified model of the devices was used to perform FE studies of the optimum design with respect to application specifications. As a first step, the $\mathrm{C}-\mathrm{V}$ curves for varying meander geometries were simulated (Fig. 13).

As expected, the pull-in voltage increases with decreasing meander length $k$, which is directly related to the spring constant of the meanders. The relation between the meander length $k$ and the spring constant for a fixed value of applied voltage is linear and ranges from $44 \mathrm{~N} / \mathrm{m}$ for $k=28 \mu \mathrm{m}$ to $35 \mathrm{~N} / \mathrm{m}$ for $k=33 \mu \mathrm{m}$. Comparisons with measurements show that the simulated value of the pull-in voltage for different meander geometries conforms very well with the measured data when using the model calibrated by the WLI measurements (maximum error $4 \%$ ).

\subsection{Fluctuations of process parameters}

The lateral film thickness of the Nb layer, which forms the bridge, undergoes fluctuations due to the deposition process. This has a significant impact on the electromechanical behavior of the capacitor. To ensure suitable behavior (no pull-in) in the specified voltage range even under these conditions, it is important to determine the relationship between the pull-in voltage and the fluctuations in film thickness. For this purpose, simulations were carried out where the film thickness of the meander suspensions on one end of the bridge was reduced by up to $20 \%$ of the original thickness. The thickness of the meander suspensions on the opposite side were kept constant. The

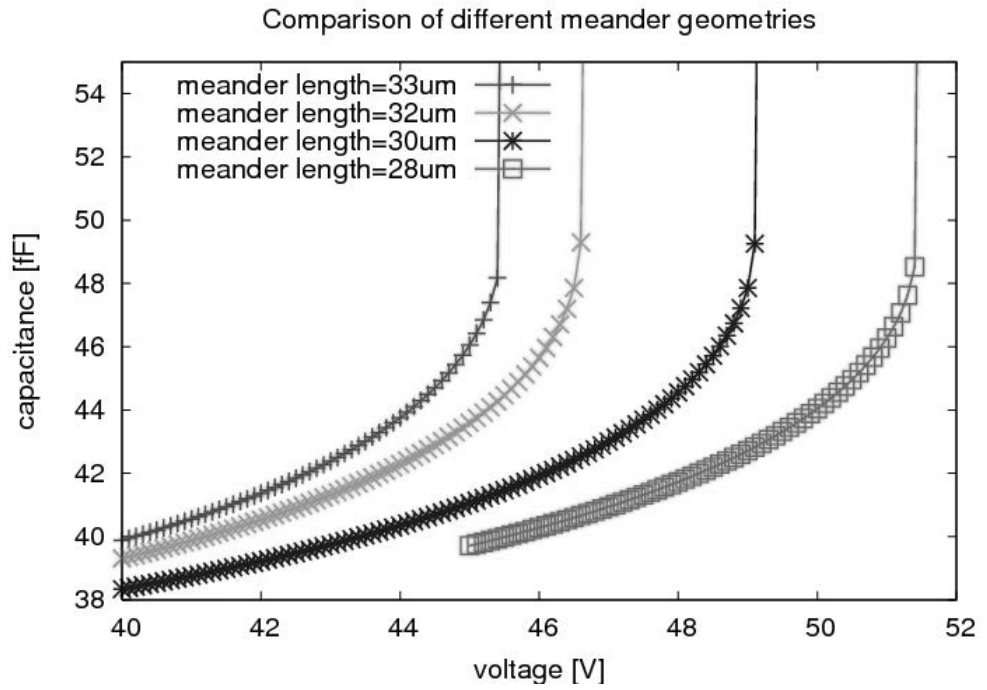

Fig. 13. Simulated C-V curves of Nb bridges with different meander lengths. 
variation of the pull-in voltage compared with the original value obtained for a constant film thickness on both sides is shown in Fig. 14.

\subsection{Eigenfrequencies}

An important characteristic quantity of a device is its natural frequency. Regarding the superconducting $\mathrm{Nb}$ varactors under investigation, it is important to avoid a resonance catastrophe caused by vibrations of the cryogenic apparatus. Therefore, the fundamental eigenfrequency was calculated by FE modal analysis for varying film thickness (Fig. 15 ) and varying meander dimensions (Fig. 16). Increasing the film thickness leads to a higher frequency as the stiffness of the device increases. The relationship is proportional, which means that increasing the thickness by $30 \%$ increases the eigenfrequency by $30 \%$.
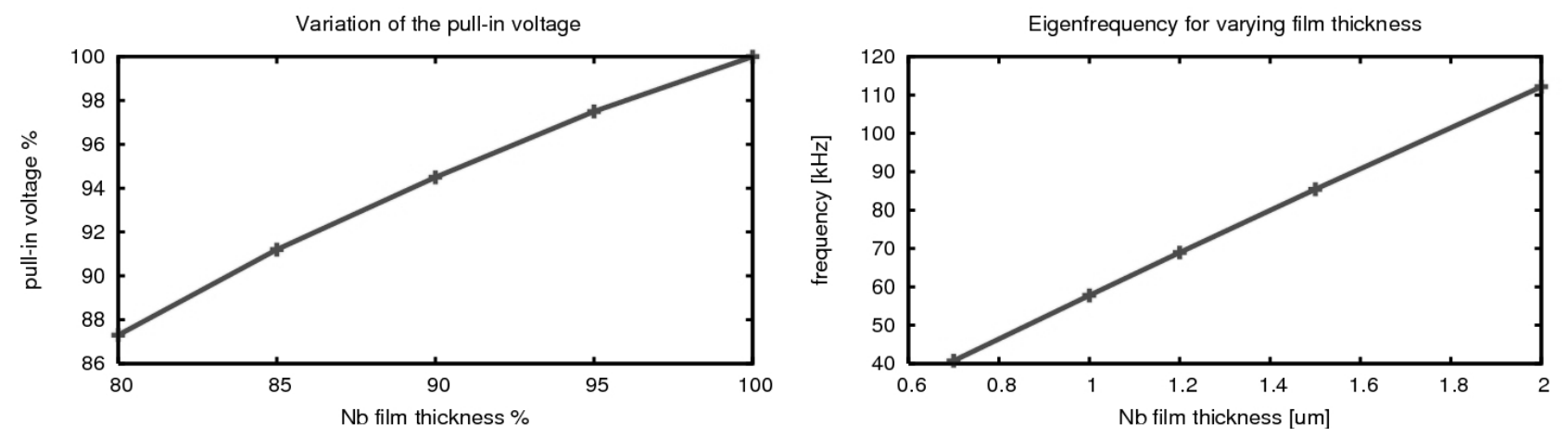

Fig. 14 (left). Variation of the pull-in voltage for reduced film thickness of meander suspensions on one side of the bridge.

Fig. 15 (right). Fundamental eigenfrequency of the capacitor vs Nb film thickness.

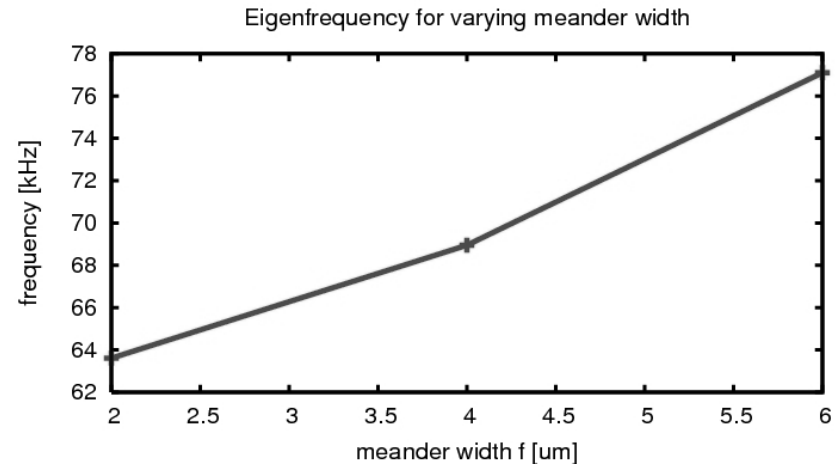

(a)

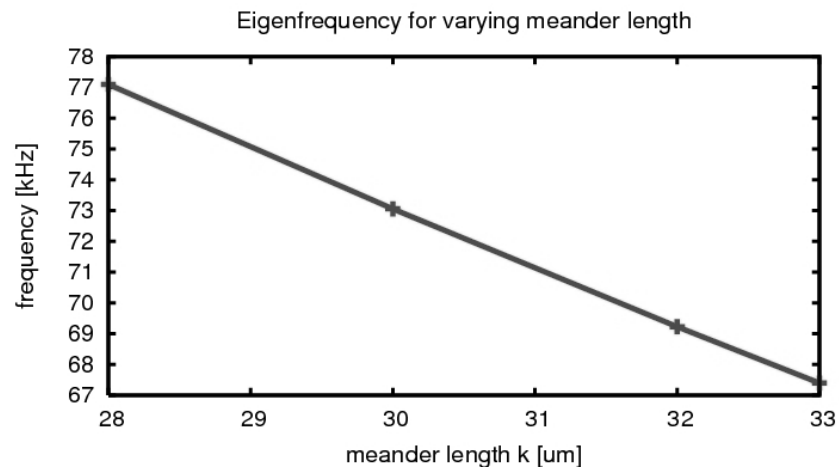

(b)

Fig. 16. Fundamental eigenfrequency of the capacitor vs meander geometry (see Fig. 3). 
The effect of the meander geometry on the eigenfrequency (Fig. 16) is a result of the change in the meander stiffness and, therefore, a change in the spring constant. Longer meanders are less stiff; thus, the eigenfrequency decreases. A change in the width $f$ of the meander has the opposite effect, since a broader meander increases the stiffness of the suspension and, therefore, the eigenfrequency of the whole structure. The width $e$ of the crossbeam of the meander has only a minimal effect on the meander stiffness, because it is much smaller than the length $k$ of the longitudinal beam.

\section{Analytical and Semianalytical Models}

Numerical simulations of 3D electromechanically coupled devices are often timeconsuming, so it is desirable to also have a relatively simple, analytical model available that allows for a quick estimation of crucial device parameters such as the pull-in voltage. Hence we attempted to obtain a realistic description of the bending behavior by an easy-to-use analytical model. Equating the mechanical and electrical forces acting on the $\mathrm{Nb}$ varactors under the constraint that the deflection of the bridge at the pull-in point is one-third of the initial gap $g_{0}$ leads to an electrical pull-in voltage $V_{\text {pin }}$ of

$$
V_{\mathrm{p} \mathrm{n}}=\sqrt{\frac{8 \mathrm{~kg}_{0}^{3}}{\mathrm{Z} \varepsilon_{0} A}}
$$

where $k$ is the spring constant of the suspension in the direction of desired motion, $g_{0}$ is the initial gap, $\varepsilon_{0}$ is the free-space permittivity, and $A$ denotes the effective area of the electrostatic forces. The crucial point here is to determine the spring constant of the suspensions. To this end, an existing analytical model of meander-shaped suspensions based on beam theory ${ }^{(11-13)}$ was extended to the case of meander springs that have varying beam widths. This analytical model was tested by comparing the analytical values of the spring constant with those extracted from numerical FE simulations for various meander geometries using quadratic shell or solid elements (see Table 2).

Unfortunately, the results of the numerical simulations show a significant discrepancy compared with the analytically calculated spring constants, whereas the simulations using different types of elements agree well with each other. This clearly indicates that the beam approximation is not applicable in this case, although the length of the longitudinal meander beams is 5 times their width. Obviously, the much shorter connecting crossbeams, which are nearly as long as they are wide, have a considerable impact on the overall spring constant. To substantiate our findings we also investigated meander geometries given in the literature ${ }^{(12)}$ by numerical FE simulations and compared our results with the analytically calculated values of the reported spring constants. Again we found large discrepancies, which clearly show that, in general, it is not justifiable to describe the elastomechanical behavior of meander-shaped suspensions using a simple beam model. Instead, a numerical model applying shell or solid finite elements should be used to obtain accurate results. Furthermore, our investigations show that the nonrigid bridge area has a considerable effect on the deflection behavior of the whole assembly, an effect that cannot easily be included in a pure analytical model. Hence, this has to be taken into account when we extract an effective spring constant for the 
Table 2

Spring constant for different meander geometries derived from numerical simulations using quadratic solid or shell elements and calculated using an analytical model based on beam theory, and the percentage deviation between the different methods.

\begin{tabular}{rrrrrr}
\hline \multicolumn{3}{c}{$\begin{array}{c}\text { Spring constants for various } \\
\text { meander geometries [N/m] }\end{array}$} & & \multicolumn{2}{c}{$\begin{array}{c}\text { Deviation between } \\
\text { different approaches [\%] }\end{array}$} \\
\cline { 2 - 3 } solid & shell & analytical model & & shell-solid & solid-analytical \\
\hline 210 & 202 & 94 & & 3.5 & 55.1 \\
168 & 164 & 80 & & 2.8 & 52.1 \\
134 & 129 & 65 & & 3.1 & 51.4 \\
105 & 102 & 57 & & 3.0 & 45.7 \\
83 & 81 & 50 & & 2.7 & 40.3 \\
75 & 73 & 46 & & 2.6 & 37.9 \\
81 & 79 & 49 & & 2.7 & 38.5 \\
94 & 92 & 57 & & 2.8 & 39.7 \\
111 & 108 & 65 & & 3.0 & 40.9 \\
58 & 56 & 36 & & 2.9 & 37.3 \\
\hline
\end{tabular}

complete device. In this case, reliable values of the effective spring constant cannot be determined without realistic numerical calculations. Here it may be helpful to split the problem into two subproblems, namely, two simple mechanical simulations, where the meander springs and bridge area are separately deflected by distinct test loads. The two separately extracted spring constants of the meander suspension $k_{\mathrm{m}}$ and the bridge $k_{\mathrm{b}}$ can then be combined to obtain an overall effective spring constant $k$ of the whole device:

$$
\begin{gathered}
k_{\mathrm{m}} / k_{\mathrm{b}}=\frac{F}{u_{z}}, \\
k=\left(\frac{1}{k_{\mathrm{m}}}+\frac{1}{k_{\mathrm{b}}}\right)^{-1} .
\end{gathered}
$$

Substituting this expression in eq. (4) gives a simple estimate of the expected pullin voltage of the bridge without full and complicated electromechanically coupled numerical simulations. Case studies verified that this approach yields an approximate pull-in voltage that is in good agreement with FE simulations as well as with measurements.

\section{Conclusions}

We derived an FE model for the optimized design of electromechanically tunable $\mathrm{Nb}$ varactors, which has been calibrated and verified with reference to WLI measurements. Although the device structure has a relatively simple geometry, suggesting that it should be amendable to allowing an analytical description in terms of compact models based on beam theory, it turns out that such models do not accurately represent the electromechanical properties of the meander-shaped suspensions of the deformable 
varactor bridge and, therefore, cannot be applied to the device investigated in this study. Furthermore, we found that the deflection of the bridge area itself has a decisive impact on the deformation behavior of the entire device; thus, the use of a numerical model is unavoidable. However, we succeeded in setting up a semianalytical model that saves computation time and allows a rapid estimation of the pull-in voltage.

A prerequisite for both the realistic electromechanical model and the simplified semianalytical model is the determination of the gap height underneath the movable bridge, which is essential for model calibration. This was achieved by WLI in combination with $\mathrm{C}-\mathrm{V}$ measurements. The optical characterization of the device revealed the existence of an internal stress gradient induced by the fabrication process which is responsible for the initial deformation of the bridges. Comparative simulations showed that this intrinsic stress does not significantly affect the electromechanical behavior of square bridges and, therefore, can be neglected in further investigations.

After model calibration, the experimental and simulated results showed good agreement and demonstrated the practicality of our model for predictive design studies. The model has been successfully used for the characterization of the dynamic behavior of a device, the effect of various mechanical parameters, and the sensitivity of device performance to fluctuations in the fabrication process. Currently work is being carried out on the further optimization of the design parameters to reduce the pull-in voltage of the devices to less than $10 \mathrm{~V}$ to find design variants that fit the specifications required for intended applications.

\section{References}

1 K. H. Gundlach and M. Schicke: Supercond. Sci. Technol. 13 (2000) R171.

2 J. Zmuidzinas and P. L. Richards: Proc. IEEE 92 (2004) 1597.

3 J. Yao: J. Micromech. Microeng. 10 (2000) R9.

4 R. Borwick, P. Stupar, J. F. DeNatale, R. Anderson and R. Erlandson: IEEE Trans. Microwave Theory Tech. 51 (2003) 315.

5 J. Zou, C. Liu and J. E. Schutt-Aine: Int. J. RF Microwave Computer-Aided Eng. 11 (2001) 322.

6 L. Dussopt and G. M. Rebeiz: IEEE Trans. Microwave Theory Tech. 51 (2003) 1247.

7 A. Abbaspour-Tamijani, L. Dussopt and G. M. Rebeiz: IEEE Trans. Microwave Theory Tech. 51 (2003) 1878.

8 G. L. Matthaei and G. L. Hey-Shipton: IEEE MTT-S Digest, 1993, LL-2.

9 M. Schicke, A. Navarrini and K. F. Schuster: Proc. Symp. Design, Test, Integration and Packaging of MEMS and MOEMS, 2005.

10 M. Schicke and K. F. Schuster: IEEE Trans. Appl. Superconductivity 13 (2003) 135.

11 G. K. Fedder: Dr. Thesis, Department of Electrical Engineering and Computer Science (University of California, Berkeley, 1994).

12 D. Peroulis, S. P. Pacheco, K. Sarabandi and L. P. B. Katehi: IEEE Trans. Microwave Theory Tech. 51 (2003) 259.

13 J. De Coster, H. A. C. Tilmans, K. Hameyer and R. Puers: Proc. 3rd Workshop on Semiconductor Sensor and Actuator Technology (SeSens2002) (Veldhoven, Netherlands, 2002) p. 4.

14 ANSYS: http://www.ansys.com.

15 M. Gyimesi and D. Ostergaard: Proc. MSM (San Juan, Puerto Rico, 1999) p. 270. 